\title{
Hábitos de uso en las Redes Sociales de los y las adolescentes: análisis de género
}

\author{
Use habits in Social Networks adolescents: Gender Analysis
}

\author{
Patricia Alonso-Ruido, Yolanda Rodríguez-Castro, María Lameiras-Fernández, Ma Victoria Carrera-Fernández \\ Facultad de Ciencias de la Educación, Universidad de Vigo
}

\begin{abstract}
Resumen
El impacto provocado por la generalización de las Redes Sociales ha supuesto drásticas transformaciones en las formas de comunicación y socialización. El objetivo de este estudio consiste en conocer los hábitos de uso en las Redes Sociales de los/as estudiantes ourensanos/as analizando las diferencias de género. La muestra estaba formada por 1283 estudiantes de la provincia de Ourense con una media de edad de 15,55 (DT: 1,6). Entre las conclusiones más relevantes se destaca que las redes sociales son utilizadas tanto por chicos como por chicas con asiduidad, no obstante se encuentran diferencias significativas en función al género en algunos aspectos. Palabras clave: redes sociales, hábitos, uso, formación.
\end{abstract}

\section{Abstract}

The impact caused by the spread of social networks has led to drastic transformations in the forms of communication and socialization. The aim of this study is to know the habits of the USO in Social Networks / as ourensanos / as students analyzing gender differences. The WAS sample of 1,283 students in the province of Ourense with a mean age of 15.55 (SD: 1.6). Among the most relevant conclusions itself stresses that social networks used son both boys regularly as for girls, but are significant differences by gender in some f: aspects

Keywords: social networks, habits, use, training.

La generalización del uso de las TICS, el boom de Internet así como el reciente impacto de las Redes Sociales han provocado un profundo impacto en las dinámicas sociales. En la actualidad, las Redes Sociales constituyen un grupo dinámico y en continua evolución, a la par que posibilitan acceder de forma sencilla al contacto con personas desconocidas (Echeburúa \& Requesens, 2012). Constituyen una de las principales fuentes de ocio para los/as adolescentes, verdaderos/as nativos/as digitales, que han encontrado en estos medios lugares en los que compartir información, establecer relaciones y comunicarse, incluso en ocasiones pueden preferir este tipo de medios al cara a cara en las conversaciones (Farber, Shafron, Hamadani, Wald, \& Nitzburg, 2012). Es por ello que, tal y como señala Gandas egui (2011), la relevancia de éstas redes ha hecho que en ocasiones pertenecer o no pertenecer a una red social deje de ser una opción para convertirse en una obligación, una necesidad para poder estar en contacto con los/as conocidos/as. Fundamentalmente porque gran parte de la vida social ya no sucede por completo en un escenario físico, revelándose la importancia del entorno virtual para los/as adolescentes y su escenario social. Esta realidad se pone de man ifiesto al observar los datos de los últimos informes y estudios (Clarke \& Montesinos, 2015; INE, 2014; Rial, Gómez, Braña, \& Varela, 2014) al respecto, que muestran cifras muy elevadas en cuanto a la tenencia y uso de las redes sociales por parte de los/as adolescentes. Así, la tenencia y uso de las redes sociales puede situar a los/as adolescentes en situaciones de vulnerabilidad e indefensión no sólo en la realidad virtual, sino también en el espacio real.

Por lo tanto, el objetivo de este estudio consiste en conocer los hábitos de uso en las Redes Sociales de los/as estudiantes ourensanos/as analizando sus diferencias en función del género.

\section{Método}

\section{Participantes}

La muestra está formada por 1.283 estudiantes del segundo ciclo de la Educación Secundaria Obligatoria $\left(3^{\circ}\right.$ y $\left.4^{\circ}\right)$ y de Bachillerato ( $1^{\circ}$ curso) de la provincia de Ourense, de los cuales un $51.1 \%$ son chicas y un $48.9 \%$ son chicos, con un rango de edad que va desde los 12 a los 25, siendo la media de edad de 15.55 (DT: 1.6). Prácticamente la totalidad de la muestra es de nacionalidad española ( $90.9 \%$ ).

El número de centros en los que se llevó a cabo la administración del cuestionario fue de seis de los ocho totales en la ciudad de Ourense (tas a participación 75\%), y nueve de los diecisiete totales en el resto de la provincia (tasa participación 53\%).

\section{Instrumentos}

Para el desarrollo de este estudio se utilizó un cuestionario creado ad-hoc, compuesto por las siguientes cuestiones: i) Cuestiones sociodemográficas: se identifican curso, nacionalidad, edad y género. ii) Cuestiones sobre la tenencia y uso de las redes sociales: hacen referencia a la tenencia de perfiles en las redes sociales, el número de contactos en estas asíco mo la sies importante en número de contactos en las redes sociales. Posteriormente se pregunta si suelen etiquetar a sus contactos en las fotos y si consideran que hacerlo los/as hace más populares socialmente. También se pregunta sobre la privacidad de sus perfiles en las redes sociales y si aceptan todas las solicitudes de amistad que reciben. A continuación se indagaba sobre la percepción de pelig ro, por un lado preguntando si consideran que tener y usar las redes sociales puede ser peligroso, respondían en una escala de 1 a 5 , siendo el 1 (nada peligroso) y el 5 (muy peligroso). Por otro lado y con la misma forma de respuesta, debían responder si consideraban que sus amistades lo consideraban peligroso o no. Por último, se consultaba sobre si recibían supervisión de sus progenitores sobre su acceso y uso a las redes sociales.ii) Formación sobre redes sociales: se les preguntó si 
alguna vez habían recibido formación sobre el uso de las redes sociales.

\section{Procedimiento}

Se contactó inicialmente con los centros vía e-mail y telefónica, informando de los objetivos y métodos del estudio. Una vez confirmada la aceptación del centro a colaborar con la investigación, se seleccionaron las aulas de E.S.O. y de Bachillerato para la aplicación de los cuestionarios.

En referencia a las cuestiones éticas se contó con colaboración y consentimiento tanto de la dirección de los centros como del profesorado (consentimiento informado pasivo).

Los/as participantes fueron informados/as de la finalidad del estudio y del carácter individual, anónimo y voluntario de los cuestionarios. El tiempo para cumplimentarlo fue aproximadamente 40 minutos.

\section{Resultados}

Entre los resultados más relevantes se destaca que las redes sociales son utilizadas tanto por chicos como por chicas con asiduidad. La mayor parte de los/as adolescentes refieren tener perfiles en una o más redes sociales (siendo Facebook, Twitter y Tuenti las más utilizadas), a las que acceden fundamentalmente a través de su móvil $\left(\mathrm{Chi}^{2}=5.86 ; \mathrm{p}<.05\right)$ tanto chicas $(88.1 \%)$ como chicos $(83.3 \%)$.

Se revelan cifras similares en lo relativo al número de contactos agregados en Facebook y Tuenti (entre 100 y 300 contactos mayoritariamente). No obstante tanto en Twitter $\left(\mathrm{Ch}^{2}=40.1 ; \mathrm{p}<.001\right) \quad$ como en Instagram $\left(\mathrm{Ch}^{2}=43.7 ; \mathrm{p}<.001\right)$ las chicas manifiestan tener más contactos que sus compañeros, entre $500(17.5 \%)$ y más de $1000(22.5 \%)$.

Son también las chicas $(27.7 \%)$ las que conceden mayor importancia al número de contactos frente a sus compañeros $(25.2 \%)$. No obstante solamente un $4.5 \%$ de ellas afirma que siempre aceptan las solicitudes de amistad aunque no sepan quién es la otra persona $\left(\mathrm{Chi}^{2}=14,53 ; \mathrm{p}<.01\right)$ frente al $9.8 \%$ de ellos que así lo afirma. (Véase Figura 1).

\section{目 Chicos 口Chicas}

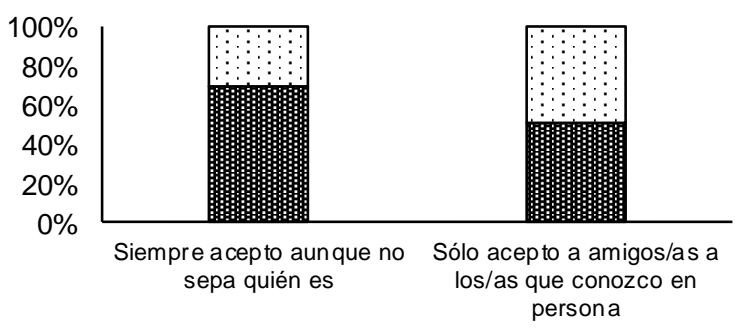

Figura 1. Aceptación solicitudes de amistad

Algunos/as de los chicos (46.5\%) y las chicas (41.6\%) que etiquetan a contactos en fotos consideran que hacerlo los hace más populares ( $23.2 \%$ chicos y $18.8 \%$ chicas $)$.

Las chicas son más cuidadosas en la privacidad de sus perfiles teniendo en mayor medida perfiles cerrados/protegidos, el $66.2 \%$ de las chicas declara que solo sus amistades pueden ver sus perfiles frente al
$56.1 \%$ de los chicos que así lo man ifiesta $\left(\mathrm{Chi}^{2}=13.61\right.$; $\mathrm{p}<.01)$. En esta línea, el $22.3 \%$ de los chicos frente al $17.9 \%$ de las chicas tienen perfiles que pueden ser observados por cualquier persona conocida y/o desconocida.

Son ellas las que tienen mayor conciencia sobre el peligro de las redes sociales, afirmando que su uso puede llegar a ser "peligroso" o "muy pe lig roso" en el $44.2 \%$ de los casos frente al $35.1 \%$ de los chicos que así lo afirma $\left(\mathrm{Chi}^{2}=23.26 ; \mathrm{p}<.001\right)$. Asimismo son ellas las que afirman en mayor medida que sus amigos/as consideran el uso de las redes sociales peligroso $(48.3 \%$ de chicos y un $52.7 \%$ de chicas).

A pesar de que tanto los chicos (91.4\%) como las chicas $(86.2 \%)$ no son supervis ados sobre el acceso y uso que hacen a las redes sociales por sus progenitores (Véase Figura 2). No obstante, son ellas las que recibir más vigilancia $\left(\mathrm{Chi}^{2}=8.44 ; \mathrm{p}<.01\right)$.

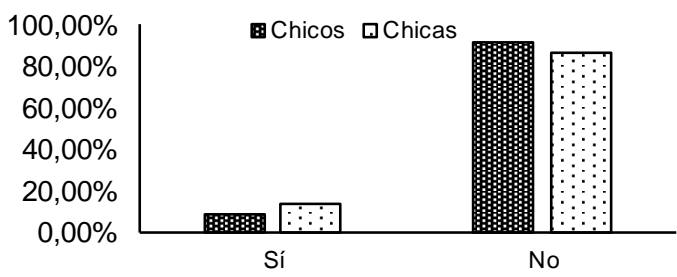

Figura 2. Supervisión redes sociales por género

Por último en lo referente a formación sobre el uso responsable de las redes sociales, a nivel general tanto chicas (76.6\%) como chicos (67\%) (Véase Tabla 1) afirman haber recibido formación; así solo un 33\% de los chicos y un $23.4 \%$ de las chicas afirman que nunca han recibido formación sobre el uso de las redes sociales $\left(\mathrm{Chi}^{2}=14,32 ; \mathrm{p}<.001\right)$.

Tabla 1.

Formación sobre redes sociales

\begin{tabular}{lcc}
\hline & Chicos & Chicas \\
\hline Sí & $67 \%$ & $76.6 \%$ \\
No & $33 \%$ & $23.4 \%$ \\
& \multicolumn{2}{c}{$\mathrm{Chi}^{2}=14.32 * * *$} \\
\hline
\end{tabular}

\section{Discusión}

En los últimos años la preocupación social sobre el impacto causado de las redes sociales e internet en los/as adolescentes se ha visto acrecentada paulatinamente. Este interés se ha volcado en numerosas investigaciones sobre el uso, la influencia de los móviles e incluso la adicción a éstos medios virtuales (Carbonell, Fúster, Chamarro, \& Oberst, 2012; Lenhart \& Madden, 2007; Purcell, 2011). En consecuencia se ha creado un clima de alarma social, sobre todo fundamentado en los peligros a los que se exponen los/as adolescentes a través del uso de Internet y de las redes sociales.

En el presente estudio se ha analizado el impacto de las redes en los/as adolescentes mediante cuestiones sobre la tenencia y uso de las redes sociales así como la 
formación que han recibido sobre el uso responsable de éstas. A nivel general se ha constatado que las redes sociales son utilizadas de forma constante tanto por chicos como por chicas, no obstante se han encontrado diferencias significativas a nivel de género en algunos de los ítems.

Entre los resultados más destacables cabe destacar, en primer lugar, la constatación empírica del alto grado de implantación de las redes sociales en la muestra. Esta afirmación se sitúa en la línea de estudios similares que resaltaban el elevado nivel de implantación de éstas entre los/as adolescentes (INE, 2014; Jiménez, de Ayala, \& García, 2013; Rial, Gómez, Braña, \& Varela, 2014; Valedor do Pobo, 2011, 2014).

En segundo lugar, la red social Facebook, cobra especial importancia al ser la que más utilizan los/as adolescentes, no obstante esta red fue también encontrada como la más utilizada en otros estudios (Almansa, Fonseca, Castillo, 2013). A nivel general, en todas las redes sociales tanto chicas como chicos refieren tener cifras verdaderamente elevadas de "amigos/as", observándose diferencias significativas en redes como Twitter o Instagram, en donde las chicas tienen cantidades mucho más elevadas. En relación a esto un estudio (Estébanez \& Vázquez, 2013) sobre la desigualdad de género y el sexis mo en las redes sociales se demuestra que las chicas suelen hablar con mayor frecuencia de sus sentimientos y frustraciones, por lo que se exponen de una manera más íntima en la red.

De esta forma se pone de manifiesto que el uso de las redes sociales es en la actualidad una actividad habitual en el desarrollo social de los/as adolescentes (Lázaro, Mora \& Sorzano, 2012). Han encontrado en ellas, espacios de "cibersocialización" (Pérez-Bonet. 2010) en donde compartir experiencias, hacer nuevas amistades e incluso expresar sus sentimientos o deseos sexuales (Bravo, Ramírez, \& Pons, 2013; Tabernero, Aranda, \& Sánchez, 2010). Llegando en algunos casos a aceptar solicitudes de amistad de personas desconocidas (en la línea del estudio realizado por Almansa, Fonseca, Castillo, 2013) o etiquetando a contactos en sus fotos porque consideran que los/as hace populares.

En tercer lugar, hemos detectado que acceden a Internet y a las redes sociales fundamentalmente a través de su teléfono móvil en porcentajes considerablemente más elevados que los reflejados por investigaciones anteriores (Garmendia et al., 2011). No obstante, la constante evolución de estos dispositivos electrónicos y las posibilidades actuales de éstos, pueden ser la causa de este cambio en el acceso a la Red, ya que, el reciente estudio realizado por el Valedor do Pobo en Galicia (2014) ya reflejaba esta tendencia en el acceso y uso a Internet.

En cuarto lugar, hemos evidenciado la escasa supervisión que tanto chicos como chicas tienen de sus progenitores sobre el acceso y uso que hacen a las redes sociales. Esta realidad se ha puesto de manifiesto anteriormente (Rial, Gómez, Braña, \& Varela, 2014), revelándose que el uso de Internet, es una práctica mayoritaria en edades anteriores a los 10 años (INE, 2014).
Por último, en referencia a la formación sobre el adecuado uso de las redes sociales por los/as adolescentes, nuestros resultados evidencian que en muchos casos sí refieren formación específica sobre este tema. No obstante, consideramos más adecuado un enfoque global, en la línea de lo propuesto por Finkelhor (2014). Una educación que no sería tan específica, si no que se encuadre más dentro del trabajo de las habilidades para la vida como la gestión de conflictos o el entrenamiento en habilidades sociales.

En conclusión, a la vista de los resultados obtenidos se pone de manifiesto la necesidad de formación sobre el uso responsable de las redes sociales a través de una perspectiva holística sobre las habilidades para la vida en de los y las adolescentes, así como en el colectivo docente y en las familias.

\section{Referencias}

Almansa-Martínez, A., Fonseca, O., \& Castillo-Esparcia, A. (2013). Redes sociales y jóvenes. Uso de Facebook en la juventud colombiana y española. Revista Comunicar, 40(20), 127-135. doi: 10.3916/C40-2013-03-03

Bravo, P. C., Ramírez, T. G., \& Pons, J. (2013). Juventud y redes sociales: Motivaciones y usos preferentes. Comunicar, 20 (40), 15-23

Carbonell, X., Fúster, H., Chamarro, A., \& Oberst, U. (2012). Adicción a Internet y móvil: Una revisión de estudios empíricos españoles. Papeles del Psicólogo, 33, 82-89.

Clarke, J. \& Montesinos, M. (2015). VI estudio de Redes Sociales. Madrid: Interative Advertising Bureau Spain.

Echeburúa, E. \& Requesens, A. (2012), Adicción a las redes sociales ynuevas tecnologías en niños $y$ adolescentes. Madrid: Pirámide.

Estébanez, I., Vazquez, N. (2013). La desigualdad de género y el sexismo en las redes sociales. Victoria-Gasteiz: Gazteak Bilduma

Farber, B., Shafron, G., Hamadani, J., Wald, E. \& Nitzburg, G. (2012). Children, Technology, Problems, and Preferences. Journal of Clinical Psychology: in session, 68(11), 1225-1229. doi: 10.1002/jclp.21922.

Finkelhor, D. (2014). Commentary:cause for alarm? Youth and internet risk research-a commentary on Livingstone and Smith (2014). Journal of Child Psychology and Psychiatry, 55(6), 655-658. doi: $10.1111 /$ jcpp. 12260

Gandas egui, V. D. (2011). Mitos y realidades de las redes sociales. Prisma Social: revista de ciencias sociales, (6), 340-366.

Garmendia, M., Garitaonandia, C., Martínez, G., \&Casado, M. A. (2011). Riesgos y seguridad en internet: Los menores españoles en el contexto europeo. Universidad del País Vasco, Bilbao: EU Kids Online. Recuperado http://www.sociologia.ehu.es/s0018eukidsct/es/contenid os/noticia/eukids_informe_280311/es_not/adjuntos/Info rme_Espa\%C3\%B̈1a_completo_red.pdf

INE. (2014). Encuesta sobre Equipamiento y Uso de Tecnologías de Información y Comunicación en los 
Hogares, Año 2014. Recuperado de http://www.ine.es/prensa/np864.pdf.

Jiménez, A. G., de Ayala, M. C. L., \& García, B. C. (2013). Hábitos de uso en Internet y en las redes sociales de los adolescentes españoles. Comunicar, 21(41), 195-204

Lázaro, I., Mora, N., \& Sorzano, C. (2012). Menores y Nuevas Tecnologías. Madrid: Tecnos.

Lenhart, A. \& Madden, M. (2007). Social Networking Websites and Teens: An Overview. Recuperado de http://www.pewinternet.org/2007/01/07/social-networki ng-websites-and-teens.

Pérez-Bonet, G. (2010). Cibersocialización y adolescencia: un nuevo binomio para la reflexión en educación social. Revista de Educación Social,11. Recuperado de

http://www.eduso.net/res/?b=14\&c=129\&n=367.

Purcell, K. (2011). Trends in Teen Communication and Social Media Use. Recuperado de http://www.pewinternet.org/Pres entations/2011/Feb/PIP -Girl-Scout-Webinar.aspx (13-04-2012)

Rial, A., Gómez, P., Braña, T., \& Varela, J. (2014). Actitudes, percepciones y uso de Internet y las redes sociales entre los adolescentes de la comunidad gallega (España). Anales de Psicología, 30(2), 642-655. doi: 10.6018/analesps.30.2.159111.
Tabernero, C., Aranda, D., \& Sánchez, J. (2010). Juventud y tecnologías digitales: espacios de ocio, participación y aprendizaje. Revista de estudios de juventud, 88, 77-96.

Valedor do Pobo de Galicia. (2014). Informe Adolescentes e Internet en Galicia: "Mocidade on line" en Galicia. Recuperado de http://des cargas.valedordopobo.com/index.php?s=156\& $\mathrm{i}=136$.

\section{Agradecimientos}

A todos los centros educativos de la provincia de Ourense que han participado en la investigación, a sus directores/as y equipos docentes por su interés y buena disposición. También a los/as adolescentes objeto de análisis en la presente investigación por su tiempo y opiniones. 